INPLASY

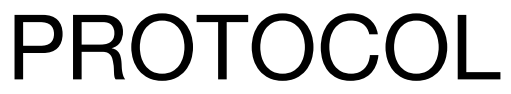

To cite: Yue et al. Analysis of risk factors for complications of perforator propeller flap used for soft tissue reconstruction after malignant tumor resection: A systematic review and meta-analysis. Inplasy protocol 202170070. doi:

10.37766/inplasy2021.7.0070

Received: 22 July 2021

Published: 22 July 2021

Corresponding author:

Shuai Yue

xhzyys2021@gmail.com

Author Affiliation:

Department of Plastic and

Reconstructive Surgery,

Plastic Surgery Hospital, Chinese Academy of Medical Sciences and Peking Union Medical College.

Support: None.

Review Stage at time of this submission: Preliminary searches.

Conflicts of interest:

None declared.

\section{Analysis of risk factors for complications of perforator propeller flap used for soft tissue reconstruction after malignant tumor resection: A systematic review and meta-analysis}

Yue, S1ㄱ Ju, MR2; Su, Z33.

Review question / Objective: We are going to utilize a metaanalysis to explore the risk factors of using the perforator propeller flap to reconstruct defects after malignant tumor resection. For this, we will perform a systematic review of the literature and conducted a meta-analysis of published data to identify the significant risk factors for complications or failure of the perforator propeller flap. Additionally, we will provide several recommendations for patient selection based on this analysis.

Information sources: We are going to searched PubMed Central, Embase, Cochrane Library, and Medline databases for relevant articles published from January 1991 to April 2021. There are no limits on the search; if foreign language articles are located, every effort will be made to obtain English copies or translate the articles.

INPLASY registration number: This protocol was registered with the International Platform of Registered Systematic Review and Meta-Analysis Protocols (INPLASY) on 22 July 2021 and was last updated on 22 July 2021 (registration number INPLASY202170070).

\section{INTRODUCTION}

Review question / Objective: We are going to utilize a meta-analysis to explore the risk factors of using the perforator propeller flap to reconstruct defects after malignant tumor resection. For this, we will perform a systematic review of the literature and conducted a meta-analysis of published data to identify the significant risk factors 
for complications or failure of the perforator propeller flap. Additionally, we will provide several recommendations for patient selection based on this analysis.

Rationale: Perforator propeller flap has been widely used due to its numerous advantages, however, there were still various complications. Herein, we are going to analyze the risk factors for complications of perforator propeller flap used for soft tissue reconstruction after malignant tumor resection.

Condition being studied: After extensive radical treatment of malignant tumors, the wound maybe not closed directly and needs to be reconstructed by other methods. The perforator propeller flap is an ideal reconstruction method for these tumors without damaging the muscle and major vessels of the donor site. Meanwhile, the flap can be used to realize the principle of "like with like" reconstruction. However, complications and even flap loss also have been reported when the perforator propeller flap was used for defect reconstruction after malignant tumor resection. Most flap-related complications and failure result from arterial or venous insufficiency. To overcome these issues, hand-held Doppler, computed tomography angiography (CTA), and magnetic resonance angiography (MRA) have been used to detect a reliable perforator as the flap pedicle. Multifarious techniques such as near-infrared fluorescence imaging have been used to evaluate the flap perfusion. However, there are several potential risk factors in the process of patient selection, flap planning and perforator dissection, which may lead to flap complications and even flap loss. Identifying and avoiding these risk factors is critical in ensuring the success of the surgery, but few studies have examined the risk factors that may cause complications.

\section{METHODS}

Search strategy: We are going to search PubMed Central, Embase, Cochrane Library, and Medline databases for relevant articles published from January 1991 to
April 2021. This search will conduct using appropriate keywords in the English language combined with Boolean logical operators as follows: "perforator flap" OR "perforator flaps" OR "propeller flap" OR "propeller flaps" OR "perforator propeller flap" OR "perforator propeller flaps" OR "pedicled perforator flap" OR "pedicled perforator flaps" AND "neoplasm" OR "neoplasms" OR "cancer" OR "cancers" OR "malignant neoplasm" OR "malignant neoplasms" OR "neoplasm malignant" OR "neoplasms malignant" OR "malignancy" OR "malignancies".

Participant or population: All the patients who were performed perforator propeller flaps for defects reconstruction after tumor resection were included in the study.

Intervention: Risk factors that can increase the incidence of complications after the perforator propeller flap surgery. Such as age $\geq 60$, smoking, diabetes, hypertension, radiotherapy, defect located in the extremities, flap size $\geq 100$ square centimetre and rotation arc $\geq 120$ degrees.

Comparator: The patients who were performed with the perforator propeller flap for defect reconstruction after tumor resection but without the above-mentioned risk factors.

Study designs to be included: All original published studies describing the use of perforator propeller flaps in defect reconstruction after malignant tumor resection will be included.

Eligibility criteria: All original published studies describing the use of perforator propeller flaps in defect reconstruction after malignant tumor resection will be included.

Information sources: We are going to searched PubMed Central, Embase, Cochrane Library, and Medline databases for relevant articles published from January 1991 to April 2021. There are no limits on the search; if foreign language articles are located, every effort will be made to obtain English copies or translate the articles. 
Main outcome(s): Complications after the perforator propeller flaps surgery (1)venous congestion; (2)partial necrosis; (3)flap loss; (4)wound dehiscence; (5)infection; (6)fistula; (7)silicone implant contracture; (8)trapdoor.

\section{Additional outcome(s): None.}

Data management: Review manager 5.4 will be used to perform a fixed-effect metaanalysis using the inverse variance method for relative risk. Combined value will be represented using a $95 \%$ confidence interval (CI). Differences regard as statistically significant at $p<0.05$. Results of the meta-analysis will be shown using forest plots, in which horizontal lines represent confidence intervals, squares represent relative risk, and size indicate a study's weight in the meta-analysis.

Quality assessment / Risk of bias analysis: Funnel plots and Begg's test will be used to evaluate publication bias in the metaanalysis. A symmetric graph indicates that publication bias may not exist. Conversely, an asymmetric graph indicate possible publication bias or systematic difference between large-sample and small-sample studies. Begg's test with a rejection region of $p=0.05$ will be used to evaluate the existence of publication bias.

Strategy of data synthesis: Review manager 5.4 will be used to perform a fixed-effect meta-analysis using the inverse variance method for relative risk. Combined value will be represented using a 95\% confidence interval (Cl). Differences will be regarded as statistically significant at $\mathbf{p}<0.05$.

\section{Subgroup analysis: None.}

Sensitivity analysis: Sensitivity analysis will be conducted by excluding one study at a time to explore the influence of individual studies.

Language: None.

Country(ies) involved: China.
Other relevant information: None.

Keywords: Perforator flap; Propeller flap; Malignant tumor; Meta-analysis.

Dissemination plans: The meta-analysis is gong to publish in a international journals.

Contributions of each author:

Author 1 - Shuai Yue - The author will be involved in data collection, data analysis and manuscript writing.

Email: nanyiys@126.com

Author 2 - Mengran Ju - The author will be involved in data collection and manuscript writing.

Email: jumengran123@163.com

Author 3 - Zhe Su - The author will be involved in data analysis and manuscript writing.

Email: suesu0092@126.com 